OPEN ACCESS

Edited by: R. Lyle Skains,

Bournemouth University, United Kingdom

Reviewed by: Voltaire Alvarado Peterson, University of Concepcion, Chile

Md. Sayeed Al-Zaman, Jahangirnagar University, Bangladesh

${ }^{*}$ Correspondence: Ahmed Al-Rawi aalrawi@sfu.ca

tORCID:

Ahmed Al-Rawi orcid.org/0000-0002-1825-0097

Oumar Kane orcid.org/0000-0002-6843-5380

Specialty section:

This article was submitted to Science and Environmental Communication,

a section of the journal Frontiers in Communication

Received: 23 June 2021 Accepted: 13 September 2021 Published: 22 November 2021

Citation: Al-Rawi A, O'Keefe D, Kane O and Bizimana Aé-J (2021) Twitter's Fake News Discourses Around Climate

Change and Global Warming.

Front. Commun. 6:729818. doi: 10.3389/fcomm.2021.729818

\section{Twitter's Fake News Discourses Around Climate Change and Global Warming}

\author{
Ahmed Al-Rawi ${ }^{1 * t}$, Derrick O'Keefe ${ }^{1}$, Oumar Kane ${ }^{2 \dagger}$ and Aimé-Jules Bizimana ${ }^{3}$ \\ ${ }^{1}$ School of Communication, Simon Fraser University, Burnaby, BC, Canada, ${ }^{2}$ Département de Communication Sociale et \\ Publique, Faculté de Communication, Université du Québec à Montréal (UQÀM), Montreal, QC, Canada, ${ }^{3}$ Department of Social \\ Sciences at the Université de Québec en Outaouais (UQO), Gatineau, QC, Canada
}

In this empirical study, we collected about 6.8 million tweets that mentioned "fake news", and we extracted references to climate change and/or global warming to understand the public discourses around these two issues. Using a mixed method, the study's findings show that there is a clear politically polarized discussion on climate change. We found that the majority of tweets focus on the United States context though references to other Western coutnries are often made. The anti-Liberal or anti-Democratic online community was more active on Twitter than the anti-conservative or anti-Republican community. Also, more than half the examined most retweeted posts contained claims about climate change being a natural cycle or even denying it exists, while about a third of these tweets stated that climate change was anthropogenic. The implications of the study are discussed, we argue that fake news as a term has a hollow meaning as it is used as a buzzword to discredit opponents and further the political agenda of different parties not only in the United States but also in other Western countries like Australia.

Keywords: climate cahnge, fake news, global wanning, political polarization, social media

\section{INTRODUCTION}

Climate change is one important news topic where the issue of "fake news" and related phenomena plays a central role in media coverage, political debate, and the broader public discussion. As author Mark Miller notes, the scale and gravity of the climate issue provides psychological reasons for people to be open to believing news that distorts or denies reality:

Numerous scientific studies have proven that climate change is scientific fact. It is not fake news. But it is bad news. And many people don't want to face it.It's easier, for some, to believe that climate scientists are falsifying their studies so they can make money promoting the "climate change industry." For some, the fake news stories are easier to accept than the real story (Miller, 2019).

Today, various fringe groups like QAnon often regard climate change as a hoax. The latter group now boasts thousands of followers of their anonymous Internet posts that blend "hard to decipher prophecies," anti-establishment rhetoric, and frequent denunciations of mainstream news sources as fake (LaFrance, 2020) QAnon's rapid growth of influence is just one sign of the dynamic and everchanging communications and media landscape in which the notion of "fake news" is being contested and weaponized by various ideologically-motivated actors. The current global pandemic has highlighted the pivotal role that "fake news" plays in media discourse on public health and epidemiology. In the case of COVID-19 the accusations of fake news were deployed nearly every day against various media outlets by the former United States president on the defensive, demonstrating 
and accentuating the populist right's weaponization of the term to discredit mainstream journalism critical of the Trump administration. The term fake news has been harnessed as a form of networked political spamming by those aiming to attack and discredit the scientific consensus on anthropogenic climate change (Al-Rawi, 2020). Indeed, the concept of "fake news" has become today a floating signifier, a contested discursive tool, and a hollow buzzword with varied and dynamic meanings-largely due to the fact that it's a favourite expression of Donald Trump and some of his followers, who used Twitter and other social media to amplify their messages.

Especially given the powerful multiplier effect of some digital pulpits, this paper's argument is that, when it comes to media discussions of climate change, the term fake news is primarily associated with the idea that the scientific consensus, as reflected in the Intergovernmental Panel on Climate Change's (IPCC) annual reports, is a "hoax." Indeed, the intellectual and discursive tug-of-war over fake news is part of the evolution of concepts of misinformation and disinformation. The issue is extremely relevant in our ongoing time of climate change, given that allegations and counter-allegations of false information and propaganda campaigns have been central to the discursive struggle over global warming for decades. According to a recent study conducted by Brandwatch, "the climate change content that got the most engagements in the last year is from a known conspiracy site called Natural News" (Reid, 2020).

The phenomena of misinformation and disinformation with respect to climate science dates back many decades, and its role and influence on policy has been researched extensively. ExxonMobil and other major oil and gas companies, for example, have been singled out for their role in funding think tanks that specialized in casting doubt on climate science. In this way many of the companies contributing significantly to the emissions causing global warming have also "funded, shaped, and advanced climate denial" (Grasso, 2019), and this behaviour has allegedly "substantially contributed to paralysing global climate policy for decades" (Ibid.). The purpose of this web of denial has been to confuse the public and decision-makers in order to delay climate action and thereby protect fossil fuel business interests and defend libertarian, free-market conservative ideologies. (Cook et al., 2019).

This paper's argument is that, when it comes to media discussions of climate change, the term fake news is merely a tool to attack opponents. Whereas in the past the denial movement was characterized by high profile "experts" asserting their views through the lens of scientific skepticism (Jacques, 2012), the contemporary online discourse features signifiers like "fake news" far more frequently in debates about climate science.

Whether during an election campaign or not, there is now grave concern about politically-motivated groups or individuals spreading false information in order to influence or change public opinion-as well as growing concern over finanicially-motivated schemes to produce fake news articles to attract clicks (Farka and Schou, 2018: 298). Turning to the specifics of the current political debates and researches focused on "fake news," one of the hegemonic political projects, while admittedly amorphous, is the efforts of far-right political forces to promote nationalist and xenophobic political narratives, leaders, and policies. Our research into fake news and climate change is unavoidably touched by the larger global trends of the new right-wing authoritarian personified by Trump and other figures such as India's Narendra Modi and Brazil's Jair Bolsonaro. Critics of these figures argue they pose a serious threat in large part due to their aim to contribute to the "undermining of the institutions of liberal democracy” (Gandesha, 2020: 9).

\section{Literature Review}

The notion of a climate denial movement has been theorized and debated widely. Peter J. Jacques, without drawing any moral or political equivalencies, compares the phenomenon to Holocaust denial, in the sense that both represent reactionary efforts that camouflage their true intentions, sow confusion and demand "space for two competing and supposedly equally valid sides in a public debate that adherents say deserves equal treatment" (Jacques, 2012: 10). Jacques' description of the means by which climate denial is disseminated is worth considering:

“...well-credentialed contrarians serve as spokesmen (mostly men) to media forums outside peer-reviewed journals. Thus, it appears to policy elites, journalists, and of course the general public that there are two equally legitimate "sides" and that each should receive equal attention. Climate denial advocates sow confusion in a public that is often unaware that core elements of climate science have far more vetting, good-faith witnesses, corroboration, and merit (Ibid.).

It has often proven difficult for researchers to identify all the funding sources of the climate denial industry, since "many of the think tanks that continue to question established climate science are nonprofit groups that aren't required to disclose their donors" (Friedman and Tabuchi, 2019). In addition to those directly associated with fossil fuel interests, key funding and dissemination of climate denial has often been part of a broader constellation promoting rollbacks of government regulation in general. In this category, the brothers David and Charles Koch have played a key role. A recent book-length study describes Charles Koch as having created "a political influence network that is arguably the most powerful and far-reaching operation ever run out of an American CEO's office" (Leonard, 2019: 5). To give just one example, former President Barack Obama's effort to introduce climate change legislation took a circuitous route reportedly in large due to the influence of the Koch network:

Fossil fuel magnates Charles and David Koch have, through Americans for Prosperity, a conservative group they back, succeeded in persuading many members of Congress to sign a little-known pledge in which they have promised to vote against legislation relating to climate change unless it is accompanied by an equivalent amount of tax cuts. Since most solutions to the problem of greenhouse-gas emissions require costs to the polluters and the public, the pledge essentially commits those who sign to it to vote against nearly any meaningful bill regarding 
global warming, and acts as yet another roadblock to action. (Mayer, 2013).

In recent years, the scientific consensus around climate change has become more robust and the alarming effects of global warming more visible around the planet, the modalities of climate denial are changing and taking new shapes. As mentioned above, the concept of "fake news" is a floating signifier, and Donald Trump dismissed climate science outright on a number of occasions, including famously tweeting that global warming was a "hoax" perpetrated by China to advance its geopolitical interests (Worland, 2019). This issue also expalins why our study found that the majority of discourses on climate change and fake news focused on the United States context.

Prior to the explosion in the use and discussion of the phrase "fake news" in the past few years, these issues were largely discussed using the vocabulary of misinformation, disinformation, spin and propaganda. In its simplest and narrowest sense, misinformation has been defined as encompassing only unintentionally misleading content or unintentionally spread false information. Disinformation, in contrast, tends to refer to the purposeful creation of false news stories and the intentional spreading of false information (Kavanagh and Rich, 2018: 122).

Following these traditional definitions, disinformation shared unknowingly by a social media user would be a case of spreading misinformation (Farka and Schou, 2018: 298). The very architecture of social media networks, however, makes possible vast disinformation campaigns by state or non-state actors in which thousands or even millions of individual users become unwitting disseminators of misinformation. It has been argued, furthermore, that in our social media age the traditional definitions have been rendered insufficient because they neglect the increasingly-common phenomenon of users deliberately sharing information without knowing with anything close to certainty whether the information is truthful (Ross and Rivers, 2018).

Given the emergence of what author Nick Srnicek has dubbed "Platform Capitalism," in which "the digital economy is an increasingly pervasive infrastructure for the contemporary economy," the danger of large-scale disinformation campaigns is now widely discussed both by national security apparatuses and the general public (Srnicek, 2017: 5). The alleged foreign interference in the 2016 United States presidential election is only the highest profile of a number of such cases.

The concern about the spreading of misinformation is not limited to the mass proliferation of disinformation by illinformed or gullible social media users. Given the rapid growth of machine learning (artificial intelligence, or AI) and automation, it is important to look deeper than bad intentions and hoaxes to examine the threat posed by programmed, autonomous social media accounts known as "bots." "According to Woolley and Guilbeault, the political bots used in the 2016 United States elections were primarily used to create manufactured consensus" (quoted in Burkhardt, 2017: 18). The notion of "Manufacturing Consent" used to be synonymous with the media theory advanced by Noam Chomsky and colleagues that focused on the "filters" and biases of mainstream, corporateowned media outlets (Chomsky and Herman, 1998). The media landscape is now much more complicated and arguably much more prone to the spread of disinformation.

Whether during an election campaign or not, there is now grave concern about politically-motivated groups or individuals spreading false information in order to influence or change public opinion-as well as growing concern over finanicially-motivated schemes to produce fake news articles to attract clicks (Farka and Schou, 2018: 298). Researchers have also carried out narrower empirical studies focusing on topics such as misleading health information; while debates rage about how much people actually rely on Internet searches to self-diagnose on medical issues, the potential for physical and mental harm caused by people applying dubious, low-quality, or untrustworthy information illustrates the importance of considering the credibility of online health information in particular. (Eysenbach, 2017, p.124). Farkas and Schou also note that studies have been carried out on government-organized propaganda, hoaxes involving Wikipedia, and racist propaganda (Farka and Schou, 2018, p. 298).

\section{Theoretical Framework}

Turning to the specifics of the current political debates and researches focused on "fake news," one of the hegemonic political projects, while admittedly amorphous, is the efforts of right-wing and far-right political forces to promote nationalist and xenophobic political narratives, leaders, and policies. Among the key institutions these figures and the movements supporting them allegedly seek to undermine are media outlets that remain independent of government influence or direction, a key pillar of democratic political systems. A recent study found that Facebook was the most susceptible of the Silicon Valley digital giants to the spreading of false information (Hopp, Ferrucci, and Vargo, 2020). Of interest, researchers found that "sharing countermedia content on Facebook is positively associated with ideological extremity and negatively associated with trust in the mainstream news media" (Ibid.) Despite these and other findings, Facebook has faced criticism for its handling of the threat posed by disinformation. In July 2020, for instance, a number of United States Senators including Elizabeth Warren wrote to the company's executives, specifically criticizing their mechanism for fact-checking as it related to climate issues: "If Facebook is truly "committed to fighting the spread of false news on Facebook and Instagram," the company must immediately acknowledge in its fact-checking process that the climate crisis is not a matter of opinion and act to close loopholes that allow climate disinformation to spread on its platform" (Vox, 2020). Facebook has recently begun to take greater action against fake accounts accused of spreading disinformation, including disabling a number of accounts allegedly linked to employees of Bolsonaro's family members in Brazil (CNN, 2020).

Accelerating and in some ways transforming these longstanding and well-organized disinformation campaigns around climate change, the so-called "alt-right" and other powerful Internet subcultures have emerged to "take advantage of the current media ecosystem to manipulate news frames, set agendas, 
and propagate ideas" (Marwick and Lewis, 2017). Especially when analyzing a medium like Twitter, the notion of "attention hacking" is useful for understanding how far-right group have been able to "increase the visibility of their ideas through the strategic use of social media, memes, and bots-as well as by targeting journalists, bloggers, and influencers to help spread content." (Ibid.). In this contest for hegemony, Farkas and Schou identify three main uses of the concept of fake news: 1) critiques of digital capitalism, 2) critique of right-wing politics, and 3) critiques of liberal and mainstream journalism (2018).

To grasp the complexity of the signifier of Fake News and, in particular, how it is currently being shaped and reshaped by Trump, his administration, and by a diverse field of media and social media actors ranging from anonymous movements like QAnon to more traditional media commentators, or "influencers" in the parlance of YouTube and other social media platforms emerging to rival mainstream network broadcasters, we must also mention the proliferation of others terms such as "Bull Shitting" or BS. Sometimes only subtly different in form and content from conventional political spin or lies, BS is defined as being produced without any concern for the truth; it is simply made up, phony, and bogus. For this reason, BS can be considered potentially far more insidious, since it wanders so far afield of reality that it circumvents all but the most rigorous fact-checking (Berghel, 2017: 111). Other terms used to describe this and related phenomena associated in particular with the Trump administration include: "post-truth," a situation in which "objective facts are less influential in shaping public opinion than appeals to emotion" (Ross and Rivers, 2018: 11); and "alternative facts," a phrase from Trump's former spokesperson Kellyanne Conway in defence of comments by then White House press secretary which had come under fire for lack of veracity (Ibid.)

These are the constellation of discursive trends being brought to bear on the debate over climate change. As average global temperatures have risen consistently in recent years, so too have poll results showing people in the United States concerned about the threat posed by climate change. Despite the accumulation of scientific evidence and of public concern, there remains a significant portion of the population who have proven difficult or impossible to move from entrenched positions on this issue.

Perhaps the most famous studies on public attitudes towards the climate crisis are the annual surveys conducted by the Yale Program on Climate Change Communication. The most recent survey results, released at the beginning of 2020, found "(n)early six in ten (58\%) Americans are now either "Alarmed" or "Concerned" about global warming. From 2014 to 2019, the proportion of "Alarmed" nearly tripled" (Leiserowitz, Maibach, Roser-Renouf, and Smith). Notably, this was the first time in the history of the study that those who were "Alarmed" made up the largest cohort at $31 \%$. The other five categories included: concerned (26\%), cautious (16\%), disengaged (7\%), doubtful (10\%), and dismissive (10\%). (Ibid.)

These results may seem promising for those interested in seeing policies aimed at mitigating climate change implemented, but there remains a gap between the public's feelings or fears about climate change and institutional and even personal change.
Past studies, in fact, have found that better results could be achieved by appealing to other values rather than directly focusing on the scientific evidence and threats related to climate change.

In a 2012 paper arguing against the commonly-held notion "that convincing deniers that climate change is real is necessary for them to act pro-environmentally," researchers make the case that their results show that those aiming for climate mitigation policy action can potentially achieve better results by appealing to shared values that make no mention of climate science (Bain, Hornsey, Bongiorno and Jeffries, 2012). In effect they argue you can catch more flies with honey than with vinegar, as the expression goes. "Deniers are united in disbelieving in anthropogenic climate change, but many already believe that mitigation efforts can have positive effects on society" (Ibid.). In essence, they make the case for a reframing of the climate debate towards the positive societal benefits that would accrue along with mitigation efforts: "framing climate change action in terms of producing greater interpersonal warmth or societal development was more effective in promoting environmental citizenship than a frame focusing on the reality and risks of climate change, and this was particularly the case for deniers" (Ibid.). To take a simple example of a practical application, one can imagine urban planners stressing the community and neighborly benefits of city streets filled with pedestrians rather than stressing the negative consequences of car emissions for the climate.

Years before Trump and the current particular debate around social media and Fake News, Bain et al. were pointing to the way in which climate was but one part of a constellation of signifiers in a discursive and media battle: "Disturbingly for environmentalists, attitudes towards climate change and climate science seem to have become part of a constellation of attitudes defined by the "culture wars": one may have little more luck of convincing a denier that climate change is real as of convincing a conservative Christian to support abortion, or a committed liberal to oppose it" (Ibid.). We decided to conduct this study to fill a gap in literature on the discourses surrounding fake news and climate change and answer the following research questions: (RQ1) What are the main categories and political tone in the most retweeted posts referencing fake news? (RQ2) What does this suggest about the mobilization of "fake news" as a signifier in the ongoing discurive and political struggles over climate change in the United States and beyond?

\section{METHODS}

Today, culture wars and all political struggles play out in large part in cyberspace. Digital media platforms have become fiercely contested spaces, and communication research aimed in particular at the largest platforms has followed these trends. For our purposes, we used a mixed method involving different approaches. First, we used manual content analysis that includes both quantitative and qualitative measures. This is a traditional research method, and then we combined it with a digital analysis of the tweets by examining the most frequent hashtags, words, and phrases with the help of Python scripts as will be illustrated 


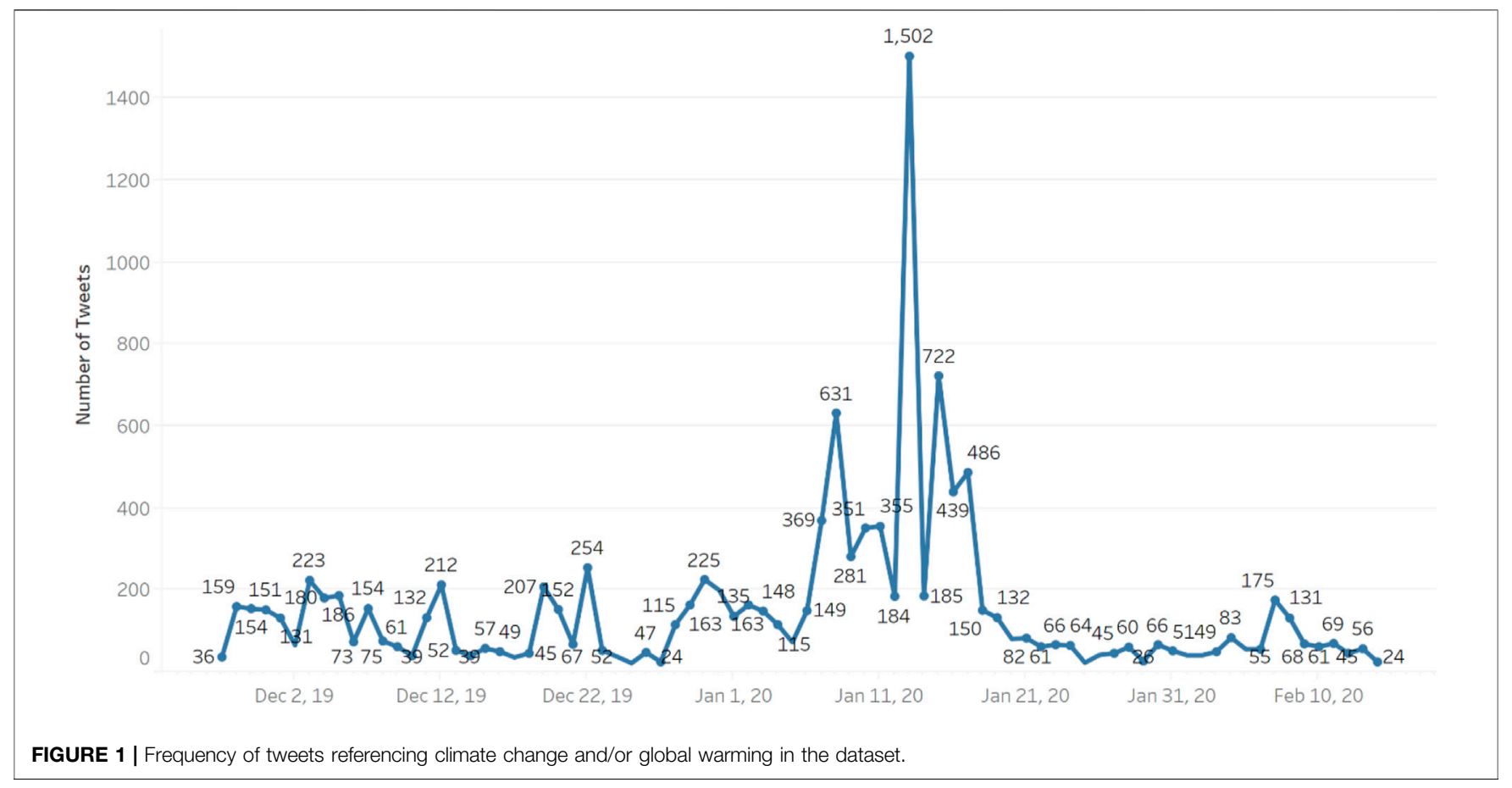

below. We followed two approaches because they complement each other and provide richer insight into the Twitter data that we examined. "Mixed method research employs both (quantitative and qualitative) approaches iteratively or simultaneously to create a research outcome stronger than either method individually" (Malina et al., 2011, p. 5).

We retrieved a recent large dataset consisting of over 6.8 million posts referencing "fake news" and/or "fakenews" pulled from Twitter using TCAT. These tweets were collected between November 27, 2019 and February 14, 2020 (See Figure 1). We started the data collection in Novemebr 2019 because it is the month Oxford Dictionaries chose the term "climate emergency" as the word of the year, and we stopped the data collection in February because of the large dataset that was collected. From this dataset, we filtered tweets that referenced "climate change" and/or "global warming", and the outcome consists of 12,055 tweets posted by 10,542 unique Twitter users. To examine our initial research question, we pulled the top 500 most retweeted posts from the filtered dataset. We found that the highest number of tweets occurred on January 13, $2020(n=1,502)$ mostly due to circulating a retweet that attacked $\mathrm{CNN}$ and defended the former President, Donald Trump, stating: "RT @adriandt31: Fake News CNN is currently doing a hit piece on @realDonaldTrump Called The Presidents Lies @CNN you are truly INSANE! All 5 of your watchers are insane as well. You stated that Climate Change helped ISIS...REALLY? REALLY? OMG your channel is TRASH! \#FakeNewsCNN". In fact, this message was retweeted 1,458 times. Following previous research (Al-Rawi, 2020; Zhou, et al., 2019), we analyzed the most retweeted posts because there is so much social media noise, and it is necessary to focus on what audiences mostly engage with which can be evident from these most retweeted posts. Two of the top five tweets, for example, simply repeat the first line of a December 2019 column published in the United Kingdom Telegraph, "Theclimate change 'emergency' is fake news." The form and content of this short, declarative sentence suggests the discursive power of the current United States president, who routinely responds to critical questions at White House briefings in similar terms, casting aside the substance of the issue by denouncing the reporter and dismissing their media outlet tout court.

This study was conducted in two stages. In the first stage, we manually coded the top 500 most retweeted posts from the dataset to look into the discursive strategies behind the Tweets aiming to support or dismiss or critique journalism reporting on the scientific consensus that climate change is caused by human activity and represents a clear and present danger and requires sustained action in response. In this regard, Badaway et al. use the methodology of text analysis on a Twitter dataset from 2016 to demonstrate the role that trolls and other partisan users based in or aligned with Russia promoted primarily conservative causes and, specifically, pro-Trump material in the run up to the United States presidential election (Badawy et al., 2018). In collecting their data, the authors relied on a massive dataset of over 43 million election-related posts, including accounts identified associated with Russian trolls. In the second stage of their research, they used label propagation to infer the ideology of all users based on the news sources they shared; this method enabled them to classify a large number of users as either "liberal" or "conservative" with a precision and recall rate of over $90 \%$ (Ibid.). We partly borrowed our content analysis method from this study and a previous one written by the authors (Al-Rawi et al., 2021) when we designed our two codebooks. In other words, we used a deductive approach in coding our dataset using 
TABLE 1 | The main categories and political tone of the most retweeted posts.

\begin{tabular}{llcc}
\hline No & \multicolumn{1}{c}{ Categories } & Frequency & Percentage \\
\hline 1 & Anthropogenic & 157 & 31.4 \\
2 & Natural cycle or not occurring & 279 & 55.8 \\
3 & Unclear & 64 & 12.8 \\
\hline \multirow{2}{*}{ No } & Political Tone & Frequency & Percentage \\
\hline 1 & Anti-Liberal or Anti-Democrat & 121 & 24.2 \\
2 & Anti-conservative or Anti-Republican & 62 & 12.4 \\
3 & Unclear target/none & 317 & 63.4 \\
\hline
\end{tabular}

Microsoft Excel. The unit of analysis is the textual content of the tweet in English language. The first codebook categorizes tweets into three types of belief with respect to climate change: 1) Anthropogenic or human-made, 2) natural cycle or not occurring, 3) unclear or vague. The second codebook, however, deals with the political tone associated with climate change discussions: 1) anti-liberal/Democratic, 2) anticonservative/Republican, and 3) political but target unclear, or not political. Two coders independently examined a sample of 50 tweets to test these two codebooks, and inter-coder reliability was satisfactory $(\alpha \geq 0.750$ for categories and $\alpha \geq 0.810$ for political tone) using Krippendorff Alpha (Krippendorff, 2011).

In making additional conclusions from the data, we used a digital method in the second stage of the research to identify the most mentioned users, top hashtags, and most used words and phrases. To achieve our goal, we used Python scripts to shed further light into the nature of climate change and fake news issues. We believe this mixed method approach, combining qualitatively analysis with quantitative digital analysis, provides important insight for both researchers and others working on the uniquely important and challenging collective action problem that is anthropogenic climate change. Especially given the medium's disproportionate number of journalist-users, understanding how the debate around fake news and climate change plays out on Twitter is key for anyone interested in media coverage of climate issues. Knowing the mechanism by which even the best journalistic efforts can be drowned in a sea of social media disinformation can help us all stay above water in the 21 st Century.

\section{RESULTS AND DISCUSSION}

To answer the study's first research question, we found that the majority $(55.8 \%)$ of the top 500 most retweeted posts were categorized as representative of the belief that climate change either isn't happening or that its causation is unrelated to human activities. The finding that varying expressions of climate change denial dominated the top tweets including the term "fake news" and climate suggests the importance of this signifier in the current political climate. Tweets that reflected behind belief in anthropogenic-caused climate change lagged far behind at $31.4 \%$ of the top 500 , while $12.8 \%$ of the tweets were ambiguous or unclear (Table 1).

Before discussing the results, it is imperative to indicate the challenges of studying social media posts around the issue of fake news and climate change due to the recurrent use of irony and the ambiguity that defines many posts. There are also challenges in interpreting social media posts, especially brief messages on a medium such as Twitter, and the understanding of which requires knowledge of local political actors and context, as well as a keen eye for irony or satire. In the following tweet, for example, one user clearly parodies those who cry "fake news" to dismiss the threat of climate change: "Climate change is a myth! \#FakeNews. The thermometers are rigged! I want a recount! Tell the penguins it's their responsibility to affect their own continent not the rest of the world!" Not all uses of irony or satire were this unsubtle, however, and this contributed to a relatively high percentage of tweets among the top 500 that the researchers had to categorize as unclear or ambiguous.

Further, a large proportion of the top tweets do not have an explicit political target, although they can be seen as part of a discursive struggle linked to political actors. The phrase and its primary usage as a term to dismiss countervailing evidence or reports one does not agree with coming from mainstream news sources, lends itself to short pithy phrases. One tweet, for instance, is characteristic of the many posts that dismiss climate science without a clear political actor being targeted: "Because it makes better headlines to blame it on climate change. \#FakeNews." The use of the hashtag in this example, parallels the term's verbal deployment as a discursive means of rejecting the entire premise of a critical question or an issue. This usage evokes scenes in which the former President Trump used the term at White House press briefing when he wished to dismiss a question entirely.

It is important to note that nearly two-thirds of the top 500 tweets do not have a clear political intent which is partly a function of the nature of the medium. Several of the top tweets, for example, contain merely the name of a newspaper columnist and a headline, "GOLDSTEIN: Political promises on climate change are fake news." This is an example of a tweet which we categorized as unclear both in terms of its characterization of climate change and its political intent. The headline points to a more sophisticated usage of the fake news signifier, and we can suppose that the columnist may not directly be questioning the scientific consensus but rather casting doubt on the political feasibility of promises around mitigation. To make any definite conclusions, however, would require analysing the full article.

In general, the findings of this study confirm that former United States President Trump and his singular reach and influence on Twitter play a central role in amplifying the term "fake news" and its discursive and polarizing power with respect to the climate debate. The most-retweeted post, for example, comes from a user who tags Trump's account in his denunciation of CNN, concluding with, "You stated that Climate Change helped ISIS ... Really? Really? OMG your channel is TRASH! \#FakeNewsCNN." Our findings on the most mentioned users also show clear political polarization. For instance, the most mentioned user in the entire dataset is United States President Trump $(n=1,629)$-followed closely by CNN $(n=1,596)$.

Here, we can surmise that CNN's segment included reference to the frequently-cited notion that droughts potentially made 
TABLE 2 | The top 50 most mentioned hashtags.

\begin{tabular}{|c|c|c|c|c|c|}
\hline No & Hashtags & Count & No & Hashtags & Count \\
\hline 1 & fakenews & 2045 & 26 & realfood & 40 \\
\hline 2 & fakenewscnn & 1,462 & 27 & cop25 & 37 \\
\hline 3 & wearethenews & 231 & 28 & india & 34 \\
\hline 4 & climatechange & 149 & 29 & wwg1wga & 31 \\
\hline 5 & climatehoax & 138 & 30 & coronavirus & 31 \\
\hline 6 & cdnpoli & 138 & 31 & bushfiresaustralia & 28 \\
\hline 7 & globalwarming & 106 & 32 & climatechangehoax & 27 \\
\hline 8 & auspol & 100 & 33 & kag & 26 \\
\hline 9 & arsonemergency & 96 & 34 & maga & 25 \\
\hline 10 & ableg & 94 & 35 & trump & 25 \\
\hline 11 & abpoli & 93 & 36 & presidenttrump & 24 \\
\hline 12 & bushfirecrisisaustralia & 92 & 37 & detoxthealgorithm & 24 \\
\hline 13 & onevoice1 & 83 & 38 & yqrec & 23 \\
\hline 14 & savetheearth & 68 & 39 & ge2020 & 23 \\
\hline 15 & weallcandosomethingaboutit & 68 & 40 & climateaction & 23 \\
\hline 16 & startnow & 68 & 41 & yqr & 23 \\
\hline 17 & vicfallsisnotdry & 57 & 42 & resist & 23 \\
\hline 18 & fake & 57 & 43 & snp & 23 \\
\hline 19 & fakeclimate & 53 & 44 & australianbushfiresdisaster & 22 \\
\hline 20 & fakeoutrage & 53 & 45 & dublincentral & 22 \\
\hline 21 & climateemergency & 50 & 46 & boycottmurdoch & 21 \\
\hline 22 & fakepresident & 50 & 47 & theirabc & 21 \\
\hline 23 & illegitimatepresident & 50 & 48 & gretathunberg & 20 \\
\hline 24 & australianfires & 41 & 49 & bushfireaustralia & 20 \\
\hline 25 & Ichf & 40 & 50 & australiaonfire & 20 \\
\hline
\end{tabular}

worse by climate change in the Middle East assisted the outbreak and severity of Syria's civil war, which in turn contributed to the expansion of ISIS (Wilson, 2017). The evidence of this sort of chain of causation, however, is largely beside the point, as tweets like this one point to the notion that climate change is often interpreted as part of a constellation of signifiers, eliciting a basically emotionally response in which the allegation of "fake news" serves as a way of dismissing the substance of a media report out of hand.

Our digital method investigation of the entire dataset shows similar findings regarding the focus on $\mathrm{CNN}$ and liberals. For instance, Table 2 lists the most used hashtags, and we can see that the second top hashtag is \#fakenewscnn $(n=1,462)$, and the word $\mathrm{CNN}$ is also the $5^{\text {th }}$ most used word $(n=3,202)$ followed by many associated negative terms often used against liberals such as "lies" $(n=1,664)$, "liar" $(n=1,365)$, "arson" $(n=1,364)$, "disgusting” $(n=685)$, "hoax" $(n=562)$, and "arsonists" $(n=445)$. For example, one of the popular retweeted posts states: “@CNN Who's behind the fire's pushing climate change lies CNN???? Fake news". The examination of trigrams (three words together) also shows similar results as the top phrase in the list is "fake news CNN" ( $n=1,508)$ followed by similar ones like "channel trash fakenewscnn" ( $n=1,459)$ "arson climate change" $(n=698)$, "disgusting democrats always" $(n=676)$. Though some of the most recurrent hashtags and words carry a clear anti-liberal tone, Table 2 also shows a few opposite hashtags that are often used by the anti-Republican community in their attacks against Trump and conservative media such as \#fakepresident, \#illegitimatepresident, and \#boycottmurdoch.

To answer the second research question, the results illustrate the term's importance beyond, yet in connection with, the context of United States politics. Many of the most-retweeted posts refer to the massive Australia wildfires of 2019-2020, often now referred to as the Black Summer, and debates over the fires' causation and relationship to climate change. For instance, one tweet condemns United States Democratic Congresswomen Alexandria Ocasio-Cortez for linking the Australian fires with climate change, declaring, "It was ARSON! Not climate change! She is a liar!" This is an example of a common phenomenon: a significant number of the top tweets connected denying of human agency in climate change with political attacks against liberal, Democratic or left-of-center political actors, and the recurrent phrase cited above on "disgusting democrats" is one example of this form of polarization. Our digital method investigation of the top most mentioned users show that some famous politicians are found in the top 20 list including the United States Congresswoman Alexandria Ocasio-Cortez who is the 5th and 8th most mentioned user (@RepAOC and @AOC) $(n=1,403)$. References to other politicians include Hillary Clinton $(n=104)$ and the Australian Prime Minister Scott Morrison, while a few other liberal media outlets are also included in the top mentioned users like the Telegraph $(n=1,078)$ and Global News $(n=230)$.

In carrying out this analysis, attention to national context was important. In Australia, for instance, the government led by Liberal Party prime minister Scott Morrison is largely viewed as conservative and often associated with denial about the severity of climate change. Although United States political categories often dominate the polarized debate online over climate change, the precise meaning of terms vary greatly across jurisdictions. For instance, Table 2 shows one common hashtag which is "Where We Go One We Go All" or wwglwga $(n=31)$ that is frequently used by the far-right conspiracy group called QAnon. 
Unsurprisingly, given that climate change denial accounted for a majority of the top 500 retweets, our research identified fully twice as much anti-liberal or Democratic (24.2\%) political intent in the tweets as anti-conservative or Republican (12.4\%), while the remaining $63.4 \%$ of the top 500 tweets were classified either as unclear or as having no specific political target. As noted, specific attacks on Democratic or left-of-center political actors were often paired with tweets dismissing climate change as "fake news." Characteristic of this type is the following tweet response to former Democratic presidential candidate Hillary Clinton: "Fake news Not climate change 200 Arsonists." Table 2 also lists some of the recurrent hashtags used by this community such as \#climatehoax, \# fakeclimate, and \#climatechangehoax.

Similarly, though less frequently, advocates of tougher action to mitigate climate change sometimes pair their analysis of "fake news" with an explicit political message. For instance, one user states, "Tory idiot Heather Wheeler has been feeding a fake claim that arsonists are behind the Australian fires. Fake news spread by climate change deniers." Again, Table 2 lists some of the common hashtags that this community, which often attacks anti-climate change deniers, often employs like \#weallcandosomethingaboutit, \#climateemergency, and \#climateaction.

In conclusion, the results indicate that the discussion around climate change and fake news is highly polarized not only in connection to the United States but also in other Western countries like Australia. The majority of the most retweeted posts had either no clear target or no political tone, but tweets carrying anti-Liberal or anti-Democratic messages were double the number of tweets that were anti-conservative or antiRepublican. The findings also show that over half the most retweeted posts $(55.8 \%)$ claim that climate change is a natural

\section{REFERENCES}

Al-Rawi, A., Kane, O., and Bizimana, A.-J. (2021). Topic Modelling of public Twitter discourses, part bot, part active human user, on climate change and global warming. J. Environ. Media 2 (1), 31-53. doi:10.1386/ jem_00039_1

Al-Rawi, A. (2020). Kekistanis and the Meme War on Social Media. Jicw 3, 13, 2020 Available at. doi:10.21810/jicw.v3i1.2360 https://journals.lib.sfu.ca/index.php/ jicw/article/view/2360

Badawy, A., Ferrara, E., and Lerman, K. (2018). "Analyzing the Digital Traces of Political Manipulation: The 2016 Russian Interference Twitter Campaign.” 2018 IEEE/ACM International Conference on Advances in Social Networks Analysis and Mining (ASONAM), 258-265. Available at https://arxiv.org/pdf/1802. 04291.pdf.

Bain, P. G., Hornsey, M. J., Bongiorno, R., and Jeffries, C. (2012). Promoting proenvironmental action in climate change deniers. Nat. Clim Change 2, 600-603. Available at. doi:10.1038/nclimate1532 https://www.nature.com/articles/ nclimate 1532

Berghel, H. (2017). “Alt-News and Post-Truths in the 'Fake News' Era”. Computer 50 (3), 110-114. doi:10.1109/mc.2017.104

Burkhardt, C. (2017). Einfach Gehirn einschalten: Warum wir denken, wie wir denken. Libr. Tech. Rep. 53 (8), 37-60. doi:10.1007/978-3-65811188-5_2

Chomsky, N., and Herman, E. (1998). Manufacturing Consent: The Political Economy of the Mass Media. New York: Pantheon Books.

CNN (2020). Facebook shuts down network of fake accounts tied to employees of Brazil's Bolsonaro and sons. ." CNN.com, 9 July 2020. Available at: https://www. cycle or deny it is even happening, while about a third of the posts (31.4\%) claim that climate change is anthropogenic.

Future research can focus on analyzing the content of newspaper articles containing the terms "fake news" and "climate change" which would likely turn up more complementary results in terms of seeing how proponents of different political agendas are deploying these terms. However, analysis of large social media datasets like this one can serve as a guide for further media research and analysis especially during the COVID-19 pandemic.

\section{DATA AVAILABILITY STATEMENT}

The original contributions presented in the study are publicly available. This data can be found here: https://doi.org/10.6084/ m9.figshare.16574807.v1.

\section{AUTHOR CONTRIBUTIONS}

All authors listed have made a substantial, direct, and intellectual contribution to the work and approved it for publication.

\section{ACKNOWLEDGMENTS}

We would like to thank the Social Sciences and Humanities Research Council (SSHRC) (grant \# 430-2018-0769) which funded the research project "Climate Change and Digital Media. A mixed topography of the hashtag \#climatechange on Twitter". This paper is one the outcomes of this research project.

cnn.com/2020/07/09/americas/brazil-bolsonaro-facebook-fake-accounts-intl/ index.html.

Cook, J., Supran, G., Lewandowsky, S., Oreskes, N., and Maibach, E. (2019). America Misled: How the fossil fuel industry deliberately misled Americans about climate change. Fairfax, VA: George Mason University Center for Climate Change Communication. Available at https://www. climatechangecommunication.org/america-misled/.

Eysenbach, G. (2017). "Credibility of Health Information and Digital Media: New Perspectives and Implications for Youth," in Digital Media, Youth, and Credibility. Editors M. J. Metzger and J. Andrew (Cambridge, MA: MacArthur Foundation Series on Digital Media and Learning The MIT Press), 123-154. Available at https://tspace.library.utoronto.ca/bitstream/ 1807/9906/6/Eysenbach2007.pdf.

Farka, J., and Schou, J. (2018). Fake News as a Floating Signifier: Hegemony, Antagonism and the Politics of Falsehood. J. Eur. Inst. Commun. Cult. 25, 3. doi:10.1080/13183222.2018.1463047

Friedman, L., and Tabuchi, H. (2019). Following the Money that Undermines Climate Science. New York, NY: New York Times. Available at https://www. nytimes.com/2019/07/10/climate/nyt-climate-newsletter-cei.html.

Gandesha, S. (2020). Spectres of Fascism: Historical, Theoretical and International Perspectives. London: Pluto Press.

Grasso, M. (2019). Oily Politics: A Critical Assessment of the Oil and Gas Industry's Contribution to Climate Change. Energy Res. Soc. Sci. 50, 106-115. doi:10.1016/j.erss.2018.11.017

Hall, S. (2015). Exxon knew about Climate Change almost 40 years ago. Scientific American. 25 October 2015. Available at https://www. scientificamerican.com/article/exxon-knew-about-climate-changealmost-40-years-ago/. 
Hopp, T., Ferrucci, P., and Vargo, C. J. (2020). "Why Do People Share Ideologically Extreme, False, and Misleading Content on Social Media," in A Self-Report and Trace Data-Based Analysis of Countermedia Content Dissemination on Facebook and Twitter". Human Communication Research. May 2020. Oxford University Press. Available at https://academic.oup.com/hcr/articleabstract/doi/10.1093/hcr/hqz022/5840447?redirectedFrom=fulltext.

Jacques, P. J. (2012). A general theory of climate denial. Glob. Environ. Polit., Vol. 2. MIT Press. Available at. doi:10.1162/glep_a_00105 https://www. mitpressjournals.org/doi/abs/10.1162/GLEP_a_00105

Kavanagh, J., and Rich, M. D. (2018). Truth Decay: An Initial Exploration of the Diminishing Role of Facts and Analysis in American Public Life. California: RAND Corporation.

Krippendorff, K. (2011). Computing Krippendorff's Alpha-Reliability. Retrieved from http://repository.upenn.edu/asc_papers/43.

LaFrance, A. (2020). “The Prophecies of Q." the Atlantic. June 2020. Available at https:/www.theatlantic.com/magazine/archive/2020/06/qanon-nothing-canstop-what-is-coming/610567/.

Leonard, C. (2019). Kochland: The Secret History of Koch Industries and Corporate Power in America. Simon: Simon \& Schuster.

Malina, M. A., Nørreklit, H. S. O., and Selto, F. H. (2011). Lessons learned: advantages and disadvantages of Mixed Method research. Qual. Res Acc Man 8 (1), 59-71. doi:10.1108/11766091111124702

Marwick, A., and Lewis, R. (2017). Media Manipulation and Disinformation Online. Data and Society, 15 May 2017. Available at https://datasociety.net/ library/media-manipulation-and-disinfo-online/.

Mayer, J. (2013). “Koch pledge tied to Congressional climate inaction." New Yorker. 30 June 2013. Available at https://www.newyorker.com/news/news-desk/kochpledge-tied-to-congressional-climate-inaction.

Miller, M. (2019). Fake News: Separating Truth from Fiction. Minneapolis: Lerner Publishing Group.
Reid, L. (2020). "Exploring the Shocking Scale of Climate Change Misinformation." Brandwatch. April 2, 2020. Available at https://www.brandwatch.com/blog/ react-climate-change-fake-news/.

Ross, S., and Rivers, D. J. (2018). “Discursive Deflection: Accusation of 'Fake News' and the Spread of Mis- and Disinformation in the Tweets of President Trump. Soc. Media + Soc. 4, 2. doi:10.1177/2056305118776010

Wilson, S. (2017). "Climate Change and the Rise of Terrorism in Northeastern Syria Samantha Wilson.” ESSAI. Spring Vol. 15. , 2017 Article 37. Available at https://dc.cod.edu/cgi/viewcontent.cgi? article=1637\&context=essai.

Worland, J. (2019). "Donald Trump called climate change a hoax. Now he's awkwardly boasting about fighting it." TIME. 8 July . Available at https:// time.com/5622374/donald-trump-climate-change-hoax-event/.

Conflict of Interest: The authors declare that the research was conducted in the absence of any commercial or financial relationships that could be construed as a potential conflict of interest.

Publisher's Note: All claims expressed in this article are solely those of the authors and do not necessarily represent those of their affiliated organizations, or those of the publisher, the editors and the reviewers. Any product that may be evaluated in this article, or claim that may be made by its manufacturer, is not guaranteed or endorsed by the publisher.

Copyright (c) 2021 Al-Rawi, O'Keefe, Kane and Bizimana. This is an open-access article distributed under the terms of the Creative Commons Attribution License (CC $B Y)$. The use, distribution or reproduction in other forums is permitted, provided the original author(s) and the copyright owner(s) are credited and that the original publication in this journal is cited, in accordance with accepted academic practice. No use, distribution or reproduction is permitted which does not comply with these terms. 